\title{
Driven oscillations of a curved object under a laminar jet of water
}

Francis Pagaud and Léa Delance*

ENS de Lyon, 69342 Lyon, France

Received: 27 September 2019 / Accepted: 3 August 2020

\begin{abstract}
By putting a ball on a flat surface under a jet of water, one may observe spontaneous oscillations of the ball of well-defined amplitude and frequency. As a simpler conformation, the study of a cylinder shows that the mere effect of the jet is sufficient to observe an oscillation for a certain range of parameters such as the curvature of the object and the characteristics of the jet. An empirical model of the forces strengthened by direct measurements of the forces and torque allowed us to predict a theoretical period of $0.64 \mathrm{~s}$ when the experimental one was $0.80 \mathrm{~s}$. Further, the origin of the oscillation was determined to be a dynamic hysteresis of the torque as it is deflected on one side of the can even when the jet hits its center. This phenomenon results in a gain of energy that counterbalances the losses by friction and leads to oscillations. Domain of oscillation is also shortly addressed while improvements of the theoretical model and other experiments are suggested as well.
\end{abstract}

Keywords: Fluid mechanics, oscillations, hysteresis.

\section{Introduction}

What do you expect when a ball is placed on a flat surface under a jet of water? Perhaps you would argue that the ball undergoes the impact of the jet and is expelled out of it. But quite surprisingly it does not: for a ping-pong ball under a jet that exits a tap, it oscillates regularly instead. The ball is covered by the liquid and is constantly brought back under the jet (Fig. 1).

The origin of this phenomenon is called the Coandă effect [1]. Indeed, when a fluid jet is brought close to a curved surface, it tends to adhere to the surface. Thus, the jet curves, which results in a deflection of the momentum of the jet perpendicularly to the surface of the object. This effect is mainly due to a difference of pressure between the two sides: the pressure near the object is lower than on the free side which causes the jet to approach the object [2-5]. As it occurs often in aeronautics or marine, the topic has been widely studied and one of its most famous applications is the levitation of a ball above a hair dryer. This latter problem is comparable to the ball oscillating under a jet because of its similar geometry. To tackle this latter issue, force measurements were conducted directly by using force sensors [6] or indirectly by using Particle Image Velocimetry [7]. This effect is however quite

\footnotetext{
*e-mail: lea.delance@espci.fr
}

qualitative and the results might be completely different depending on the type of fluid, the Reynolds number [8] or the size of the object [6,7]. However, no study tackled the issue of an oscillating ball under a jet so far. The physics of such a system would be worth understanding given that in that case an oscillation of the ball occurs.

This article aims at characterizing the oscillations, namely the amplitude and the frequency. It relies on simple and essential experimental methods for mechanics as much as possible. Good agreements between theory and experience are found while loopholes and suggestions for improvements of our model are highlighted.

\section{Methods}

\subsection{Equipment used}

The usual fluid mechanics formalism relies on the Reynolds number as a way to describe the flow. The assessment of this number in our case was the very first step to understand the problem:

$$
R e=\frac{\rho v L}{\eta} \simeq 10^{4},
$$

with $\rho$ and $\eta$ being respectively the density and dynamic viscosity of water, $L$ and $v$ the diameter and the speed 


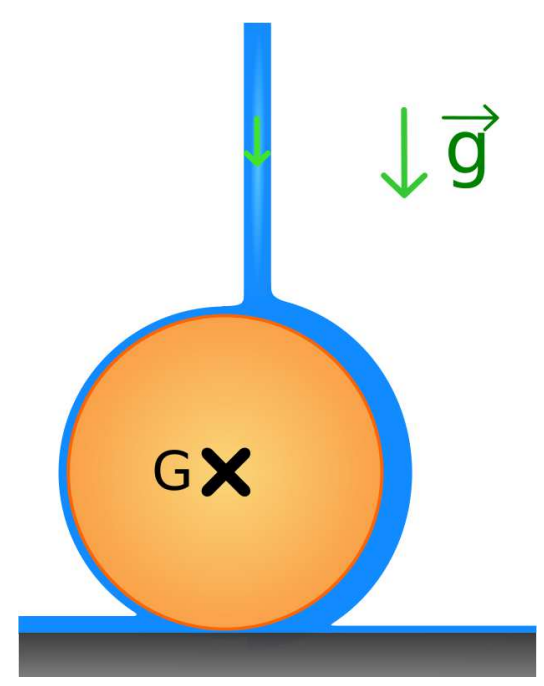

Fig. 1. Scheme of the ball under a jet of water.

of the jet before the impact. This result means that the flow of water is turbulent. As the flow out of the tap was not steady at all, a rigid nozzle was set up to prevent perturbations before the impact of the ball. Once the water meets the object, perturbations of the flow are witnessed.

In the end, the obtained laminar flow still induced spontaneous oscillations of the ball put right underneath it, even though turbulence cannot break the symmetry anymore (Supplementary Video S1). The fluctuations of the jet is not a significant phenomenon in this study.

A simpler conformation with a cylinder has been used to study the oscillations in one dimension contrary to the two dimensions of the ball on the plate. Very light cylinders like soda cans were used, weighing $m=12.4 \mathrm{~g}$ with a diameter of $D=5.8 \mathrm{~cm}$ if no further indications are provided.

Moreover, the can leaned on two perpendicular cylinders, so that the jet of water left the can and fell on the plate a few centimeters below. This conformation eliminated the interaction between the water that flows on the plate and the can that raises complex interactions such as a hydraulic jump around the ball.

What we witnessed in such a case is a regular oscillation of the can under the jet for a certain range of parameters. We can conclude that the sole influence of the jet is enough to observe the oscillations and its only effect will be investigated in the first place.

\subsection{Model of the forces applied on the can}

Before presenting our results, here is the mechanical model drawn to study the motion of the cylinder (Fig. 2). Firstly, there is the weight of the can $\vec{W}$. The force of the jet has two independent components: the drag force, parallel to the jet and written as $\overrightarrow{\boldsymbol{F}}_{\boldsymbol{y}}=-F_{\boldsymbol{y}} \overrightarrow{\boldsymbol{e}_{\boldsymbol{y}}}$ and the lift force, perpendicular to the jet and identified as $\overrightarrow{\boldsymbol{F}_{\boldsymbol{x}}}=F_{x} \overrightarrow{\boldsymbol{e}_{\boldsymbol{x}}}$, where $\overrightarrow{\boldsymbol{e}_{\boldsymbol{y}}}$ and $\overrightarrow{\boldsymbol{e}_{\boldsymbol{x}}}$ are the unitary vector along $y$ and $x$, respectively. The solid friction with the two cylinders gives also two components: the normal component $\vec{N}$, and the

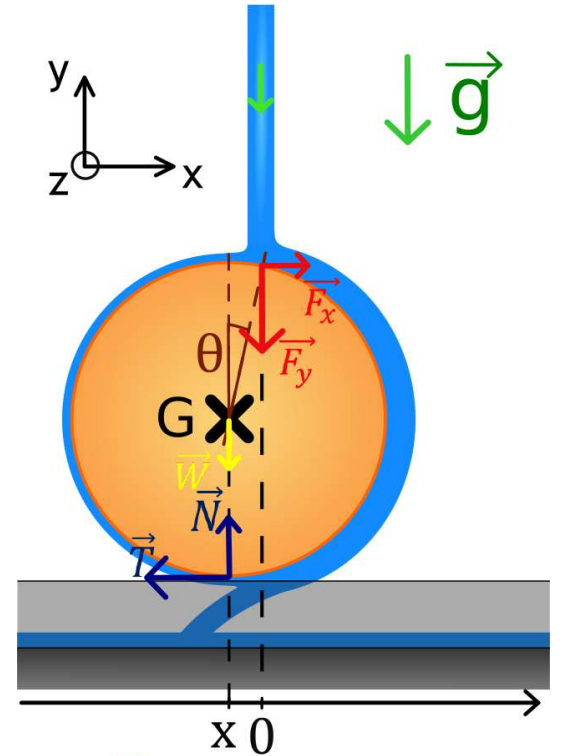

Fig. 2. Scheme of the forces applied on the cylinder. $\theta$ is the angle between the vertical and the line joining the center of the cylinder and the point of impact of the jet.

tangential one $\overrightarrow{\boldsymbol{T}}=T \overrightarrow{\boldsymbol{e}_{\boldsymbol{x}}}$. In a first assumption, the forces due to the jet are applied at the very place of impact of the jet on the can. The jet falls under the can and does not interact with the plate and the can at the same time. The angle $\theta$ is defined as the one between the vertical axis going through the center of mass $\mathrm{G}$ and the line drawn between $\mathrm{G}$ and the impact of the jet with the can.

The fundamental principle of mechanics applied on the horizontal forces and the angular momentum theorem gives the following set of equations:

$$
\left\{\begin{array}{l}
m \ddot{x}=F_{x}+T \\
J_{G} \ddot{\theta}=R T+R \cos \theta F_{x}-R \sin \theta F_{y},
\end{array}\right.
$$

with $m$ and $J_{G}$ being respectively the mass of the can and its moment of inertia along $z$ calculated in $\mathrm{G}$. The rolling hypothesis is essential as it replaces $x$ by $-R \sin \theta$, the minus sign being a matter of convention.

This model relies strongly on the assumption that the point of application of the forces is the point of impact of the jet but it is reasonable to expect these points to be close as in the impact point occurs the most drastic change in momentum.

However, for experimental reasons, we chose to take into account the torque $\Gamma$ applied on the can instead of the vertical force. The improved model is thus:

$$
\left\{\begin{array}{l}
m \ddot{x}=F_{x}+T \\
J_{G} \ddot{\theta}=R T+\Gamma .
\end{array}\right.
$$

This set of two equations contains three unknown forces. To use this model any further, the empirical expressions of the forces were investigated at first. Then the measurement of the torque was conducted. 


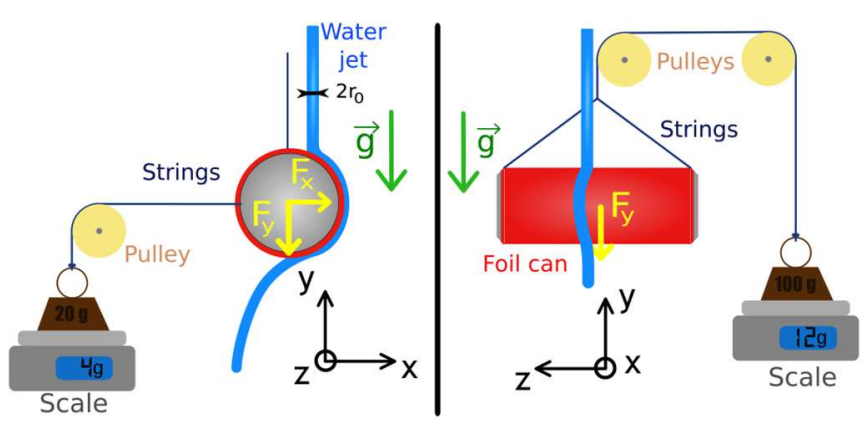

Fig. 3. Side view and front view of the setup used to measure horizontal and vertical forces applied by the jet on the cylinder.

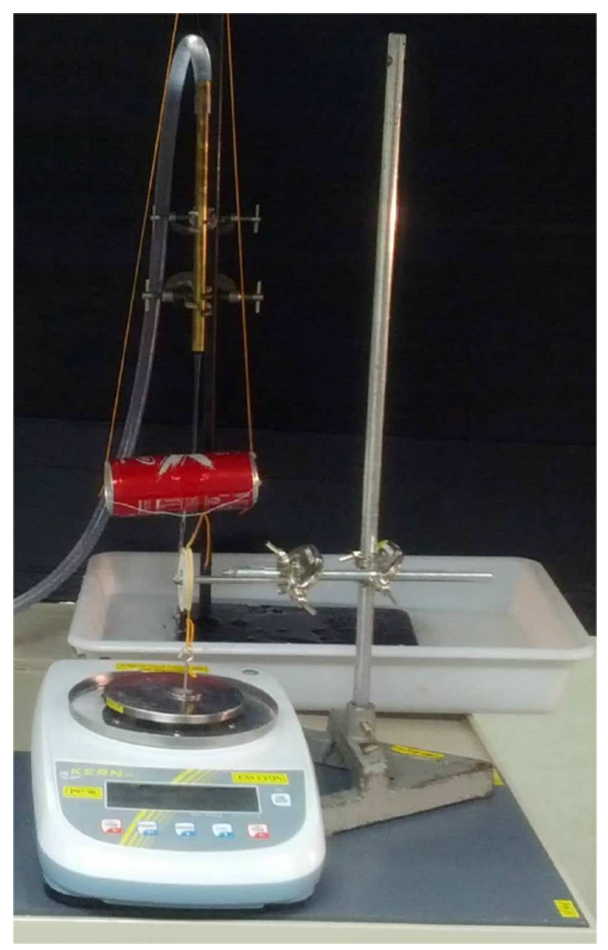

Fig. 4. Photograph of the setup used to measure the horizontal force applied by the jet.

\subsection{Measurements of the forces of the jet acting on a cylinder}

The force measurements have been carried out by using the setup depicted in Figures. 3 and 4. The cylinder was held by strings linked to masses horizontally and vertically. These masses were put on scales with a precision of $0.01 \mathrm{~g}$. The experimental setup allows us to measure the horizontal and vertical forces applied by the jet on the can.

$$
\overrightarrow{\boldsymbol{F}}+\overrightarrow{\boldsymbol{S}}+\overrightarrow{\boldsymbol{W}}=0
$$

with $\overrightarrow{\boldsymbol{F}}=\overrightarrow{\boldsymbol{F}}_{\boldsymbol{x}}+\overrightarrow{\boldsymbol{F}}_{\boldsymbol{y}}$ the horizontal and vertical forces applied by the jet on the can, $\overrightarrow{\boldsymbol{S}}=\overrightarrow{\boldsymbol{S}_{\boldsymbol{x}}}+\overrightarrow{\boldsymbol{S}_{\boldsymbol{y}}}$ the tension of both strings and $\overrightarrow{\boldsymbol{W}}$ the weight of the can. The scales were reset before the application of the jet, so

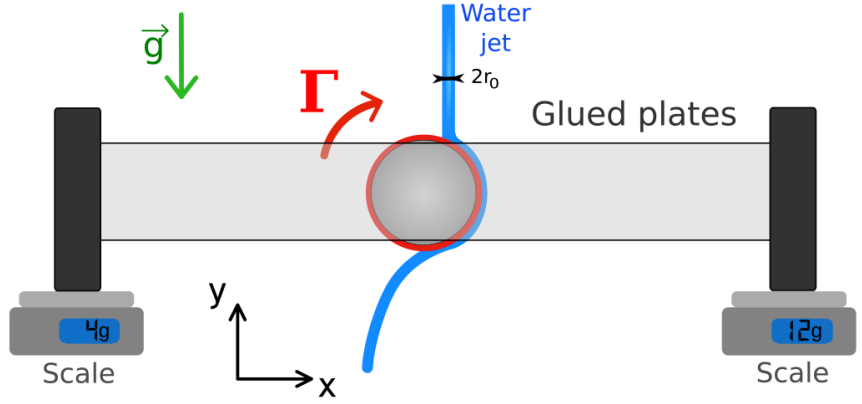

Fig. 5. Setup used for the torque measurement applied by the jet on a cylinder.

that only the forces exerted by the jet on the can will be indicated. Thus, neglecting the friction on the pulley and the string mass, the masses measured with the scales $m_{\text {meas }}$ is related to the horizontal or vertical force $F$ by: $F=m_{\text {meas }} g$ with $g=9.81 \mathrm{~m} \cdot \mathrm{s}^{-2}$. The position of the center of the jet is measured with a simple ruler, allowing to obtain the values of the forces with regard to the position of the jet.

The main limitation of this setup was the stability of the system. The jet could not be put far away from the center of the cylinder because it induced motion of the cylinder otherwise. That issue is caused by the loose strings and could not be overcome.

\subsection{Simple measurements of the torque applied to an object}

We conducted the measurements of the torque applied by the jet on the cylinder. This simple setup used is described in Figure 5. Each end of the cylinder was glued to PVC plates lying on two scales. As the lever arm $d$ between the cylinder and the scales were known, the difference of mass between the two scales leads to the measurement of the torque $\Gamma$ applied on the system. A system of rack and pinion enabled to place the jet correctly. In order to be more accurate, the jet is brought back and the scales are reset between each measurement.

Contrary to the previous experiment, this setup had no limitation due to the stability of the cylinder. The position of the jet $x$ even reached values higher than $R$, because the center of the jet, that gives the value $x$, was further than the edge of the can while the jet was thick enough to remain in contact with the can.

\subsection{Observation of the oscillating cylinder}

To conclude, the observation of the oscillating can has been carried out. We used a XIMEA camera with a welldefined framerate of 50 frames per second to observe the flow of water and measure the frequency. The can leaned on two perpendicular cylinders and was ballasted if needed with PVC disks of $5.5 \mathrm{~cm}$ in diameter and weighing up to $0.1 \mathrm{~kg}$ to study the influence of the mass. Cans were also coated with paint to witness the influence of the hydrophobicity of the surface of the can. 

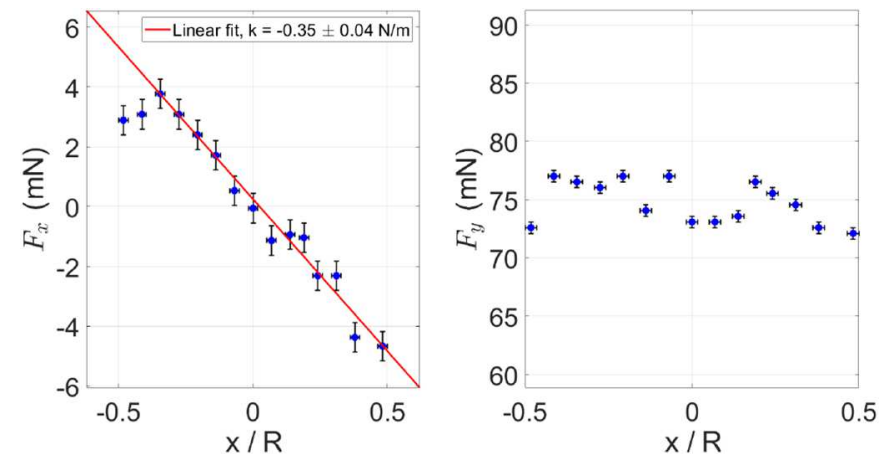

Fig. 6. Force measurements along the $x$ and $y$ axes. The characteristics of the jet are $Q=21 \mathrm{~cm}^{3} / \mathrm{s}, h=15 \mathrm{~cm}$ and $r_{0}=0.82 \mathrm{~cm}$.

\section{Results}

\subsection{Forces measurements}

The results obtained from the setup of Figure 3 are shown in Figure 6. The characteristics of the jet were fixed with a flow of $Q=21 \mathrm{~cm}^{3} / \mathrm{s}$, a height of the jet before impact with the can of $h=15 \mathrm{~cm}$ and a radius of the nozzle of $r_{0}=0.82 \mathrm{~cm}$. These values are set for every experiment unless mentioned cases. The only variable is the position of the centre of the jet. Figure 6 shows a linear force $F_{x}$ around the center of the can and a constant force $F_{y}$. Moreover, the latter force is ten times higher than the former. The expressions of $\overrightarrow{\boldsymbol{F}}_{\boldsymbol{x}}$ and $\overrightarrow{\boldsymbol{F}}_{\boldsymbol{y}}$ can thus be assimilated respectively to a spring $\overrightarrow{\boldsymbol{F}_{\boldsymbol{x}}}=-k x \overrightarrow{\boldsymbol{e}_{\boldsymbol{x}}}$ and a constant force $\overrightarrow{\boldsymbol{F}_{\boldsymbol{y}}}=-C \overrightarrow{\boldsymbol{e}_{\boldsymbol{y}}}$, with $C>0$.

However, the setup lacked stability and was not investigated any more. That is why a clear dependence of the forces on the parameters of the jet or the can was not deduced out of this setup and a measurement of the torque $\Gamma$ was carried out to complete our model.

\subsection{Torques measurements}

The setup drawn in Figure 5 was very stable and allowed to measure accurately the total torque $\Gamma$ for various cases. The results for the same parameters of the jet are displayed in Figure 7 . The torque measured is linear up to $\frac{x}{R}=0.8$ and falls to zero any further. The linear fit in red will be used in the theoretical model, so $\Gamma=-\gamma x$, with $\gamma=0.053 \mathrm{~N}$.

By replacing $\Gamma$ and $F_{x}$ by their empirical expressions in equation (3) and eliminating $T$, the resulting equation is similar to a harmonic oscillator:

$$
\ddot{\theta}+\theta \frac{R(k R+\gamma)}{J_{G}+m R^{2}}=0 .
$$

The period of oscillation ruled by this equation is

$$
T=2 \pi \sqrt{\frac{J_{G}+m R^{2}}{R(k R+\gamma)}} .
$$

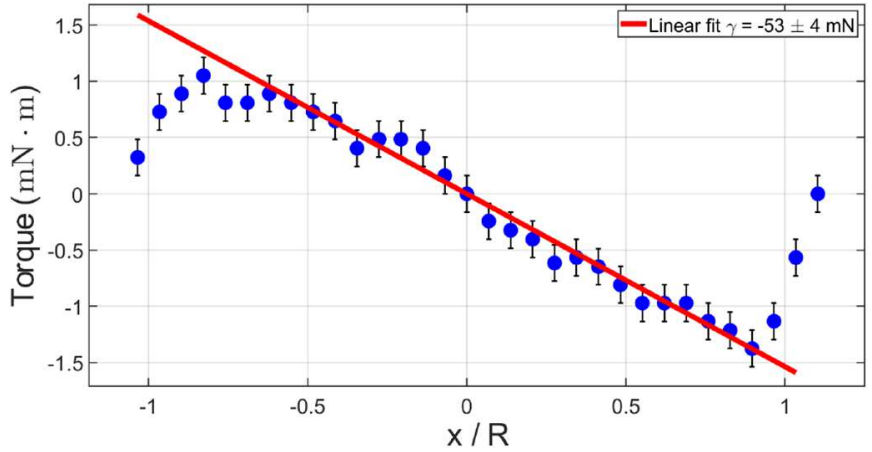

Fig. 7. Torque applied by the jet on the can depending on its relative position $\mathrm{x} / \mathrm{R}$. The experimental data are approximated to a linear relationship in red.

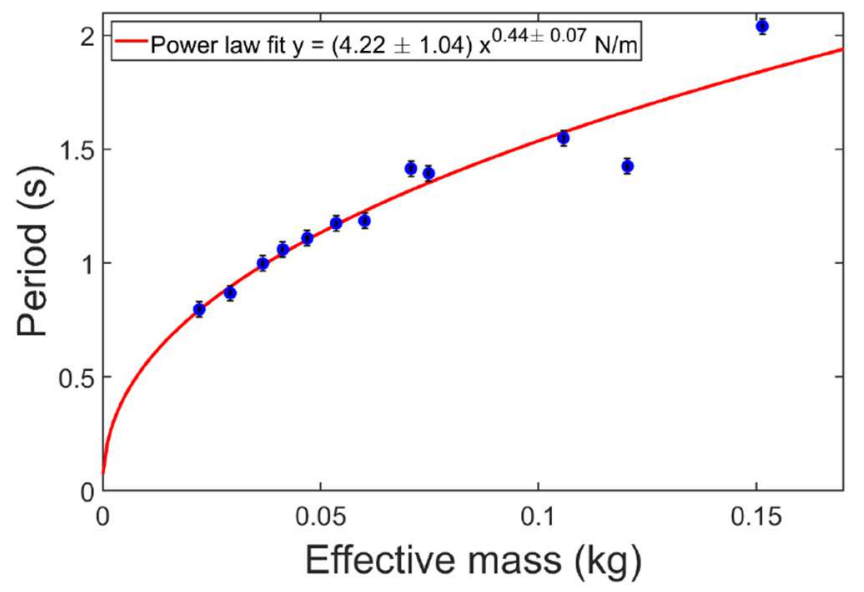

Fig. 8. Oscillation period under the jet vs effective mass of the ballasted can. The red curve corresponds to a power law fit.

The experimental agreement with this value will be addressed now.

\subsection{Frequency measurements}

Firstly, the dependence of the frequency on the effective moment of inertia was investigated. For each ballast, the moment of inertia was obtained by measuring the period of small oscillations of the ballasted can around a stick taped along the height of the can and held horizontally. This measurement could be supported by an analytic calculation of the moment of inertia. The resulting effective mass is $m_{e f f}=\frac{J_{G}+m R^{2}}{R^{2}}$.

The period of oscillation of the can under the jet is shown in Figure 8. Each time measurement corresponds to a mean over 30 oscillations. Since the jet is unchanged, the effective mass of the can is the sole variable. The observed period grows with the effective mass and the data were fitted to a power law. The fit highlighted a dependence in the effective mass to the power $0.44 \pm 0.07$. This value, close to the value 0.5 given by our model, tends to confirm it. This is strengthened by the factor 


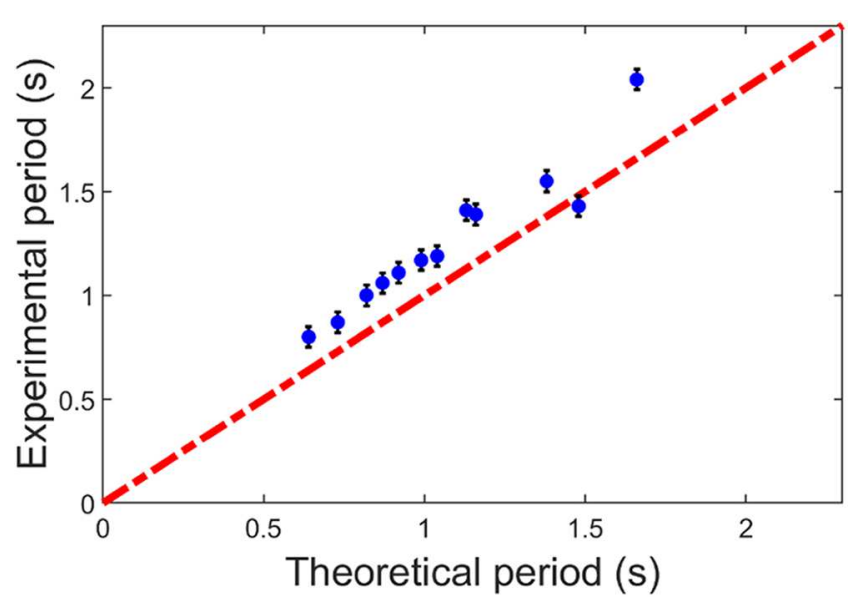

Fig. 9. Comparison of theoretical and experimental period of oscillations for ballasted cans. The straight line has a slope of 1 to compare directly theory and experiments.

$(4.22 \pm 1.04) \mathrm{s} \cdot \mathrm{kg}^{-1 / 2}$ which is similar to the theoretical one $2 \pi \sqrt{\frac{R}{k R+\gamma}}=4.29 \mathrm{~s} \cdot \mathrm{kg}^{-1 / 2}$.

Direct comparisons between the theoretical period and the measured one led to the results displayed in Figure 9. The experimental data come from Figure 8. The flow is set at $Q=21 \mathrm{~cm}^{3} / \mathrm{s}$ and the height of the jet at $15 \mathrm{~cm}$. The order of magnitude of the measured period matches with the theoretical one. However, a non-negligible discrepancy to the experimental value $\epsilon=\frac{\left|T_{t h}-T_{m}\right|}{T_{m}}$ up to $20 \%$ is witnessed, the theoretical value being almost always lower to the experimental one. This trend is also noticed when the jet parameters or the can radius are changed (results not displayed here).

\subsection{Physical origin of the oscillation}

It appeared that before reaching a stationary state with a constant and well-defined period and amplitude of oscillation, the movement of the can consists in a transient behavior during which the amplitude is amplified, whereas we would expect it to be damped due to the dissipative effects that could occurs like friction. These observations convinced us to study the physical origin of this oscillation. Our hypothesis is that the can undergoes hysteresis, providing thus a gain in energy. This would means that the torque applied by the jet on the can is different depending on whether the can comes from the right or the left.

Firstly, we investigated this problem by using the setup depicted in Figure 5 and measuring the torque in the two directions with the usual set of parameters (Fig. 10). That means that we measured the torque with the jet sweeping progressively the can from left to right (blue data) and when it goes back (from right to left, red data). This would allow to detect a static hysteresis of the torque. Figure 10 shows a shift of around $0.05 \mathrm{mN} \cdot \mathrm{m}$ in the measured torque between the two sets of data. However, this difference is very close to the error range.

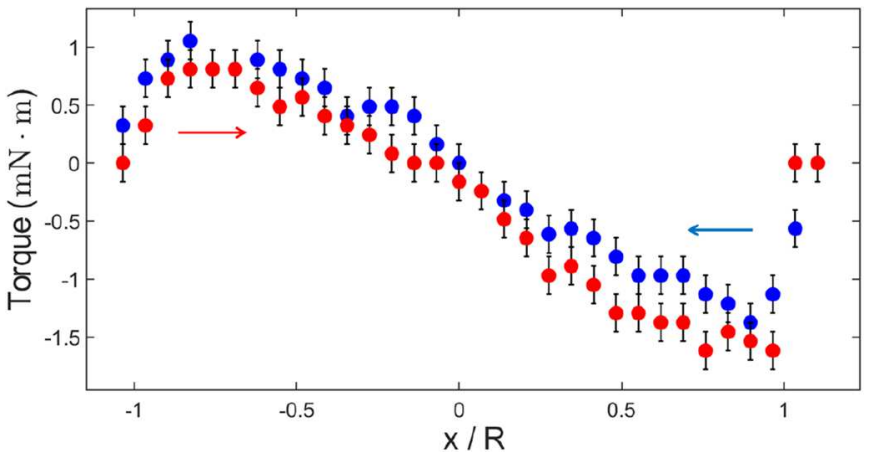

Fig. 10. Torque measurements sweeping the can from left to right and from right to left.

Moreover, this study does not take into account the motion of the can. Since torque measurements on a rotating can are hard to achieve, only a qualitative observation has been conducted in the film S2. These close-ups on the area impacted by the jet showed a delay between the position of the impact point and the deflection of the jet. In other words, the jet was still deflected on one side of the can while the impact point was on the other side. It induces a dynamic hysteresis that provides energy to the system.

The second video S3 is shot under the same conditions, except the can is coated with a hydrophilic spray. Instead of an amplification, a damping is witnessed as the jet is more symmetric around the can and no longer shows this dynamic hysteresis. This damping was also seen for a higher flow of the jet. Because of the increased section of the jet and the higher speed at the impact, the flow was deflected on both sides of the can.

\section{Discussion}

\subsection{Forces acting on the cylinder}

Measurements with different properties of the jet showed non-trivial dependence of the torque on the flow and height of the jet. As more water hits the can, the deflected momentum of the jet around the can is higher and so is the torque applied on the can. The conclusion is the same for the height of fall of water that increases the speed of the jet at the impact. So even if we were able to measure the total force and the total torque that act on the cylinder depending on the parameters of the jet, we cannot determine the contribution of the different physical phenomena at stake and of the different forces involved.

The force measurements gave an insight of the physical phenomena though. Indeed, the calculation of the contribution of the impact of the jet on the vertical force $F_{y}$ gives an upper limit by assuming that the water hits the surface and is deflected perpendicularly, and leads to $\frac{\mathrm{d} p_{\text {impact }}}{\mathrm{d} t}=\rho Q v$, with $v$ the speed of water before impact. The speed of a free jet of water falling out from a nozzle 
is theoretically known by using Bernoulli's principle [9]:

$$
v=\sqrt{\left(\frac{Q}{\pi r_{0}^{2}}\right)^{2}+2 g h},
$$

the term related to the height $h$ being prominent in our case. Its value is $v=1.72 \mathrm{~m} / \mathrm{s}$. All in all, the result is at most $\frac{\mathrm{d} p_{\text {impact }}}{\mathrm{d} t}=36 \mathrm{mN}$, which corresponds to only $50 \%$ of $F_{y}=75 \mathrm{mN}$. Thus, the contribution of the other forces on the can such as the viscosity, the capillary forces and the normal force applied by the water on the can are not negligible. Regarding this latter force, it can be approximated by considering a $0.5 \mathrm{~mm}$ thick and $3 \mathrm{~cm}$ wide layer of water around the whole can, which leads to an approximate added mass of $3 \mathrm{~g}$ and a force of $30 \mathrm{mN}$. Consequently, viscosity is likely to be the cause for the $10 \mathrm{mN}$ contribution remaining to $F_{y}$ as capillary forces are expected to be weak. This can be a consequence of the hydraulic jump around the impact of the jet, with a stream thickness $l$ lower than $0.5 \mathrm{~mm}$ and speed $w$ higher than $2 \mathrm{~m} / \mathrm{s}$. The order of magnitude of $10 \mathrm{mN}$ is reached by simply assuming a friction force similar to the one of a Hagen-Poiseuille flow on the surface $S$ around the can $F_{v i s}=\eta \frac{w}{l} S$.

\subsection{Agreement between theoretical and experimental frequencies}

The experiments show an agreement in trend between the theoretical and the experimental period of oscillation. This confirms the assumption that the torque is linear and that the interaction potential can be modeled as a harmonic potential well. However, the torque can no longer be considered as linear when the jet hits the edge of the can. The torque decreases near the edge, around $\frac{x}{R} \simeq 0.9$, until a zero torque. This is not taken into account in the model, but we can expect that a lower torque near the edge is not favourable to a quick return of the can to its equilibrium position. Hence the comprehensive theoretical period is expected to be higher because of this phenomenon, and closer to the experimental one. Simulations were carried out using the Euler method to consider the real shape of the torque, particularly the non linear part on the edge of the cylinder. Although the new theoretical period that we obtained was closer to the experimental one, it could not explain the whole discrepancy.

Indeed, it could also be due to the amount of water accumulated around the can. It has to be displaced as well as the can and a change of the effective mass used in the model should be considered. This leads to an added mass of around $3 \mathrm{~g}$. This mass being far away from the axis of symmetry of the can, the added effective mass is doubled because of the moment of inertia. It is not negligible before the low effective mass of the soda can of $22 \mathrm{~g}$. When taken into account, the theoretical period goes from $0.64 \mathrm{~s}$ to $0.72 \mathrm{~s}$, much closer to the experimental value of $0.80 \mathrm{~s}$. Unfortunately no experiment was carried out to measure the actual mass of water around the can for each experiment. Yet this could significantly improve
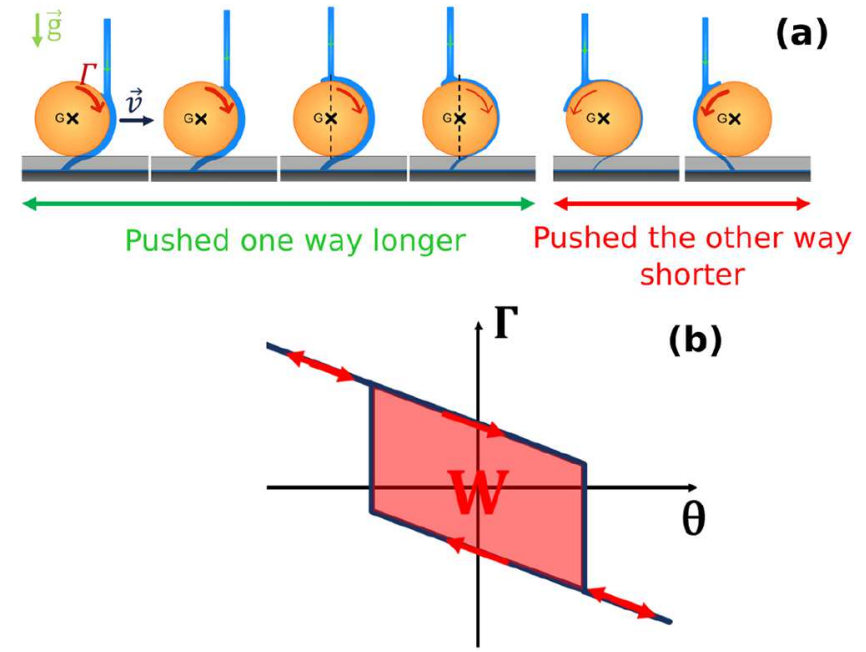

Fig. 11. (a) Scheme of the dynamic hysteresis responsible for the amplification of the amplitude of oscillation: the torque in the first direction (green) lasts longer than in the other (red). (b) It results in a modified profile of $F_{x}$ represented schematically by the blue line. The amount of energy brought to the system $W$ is the red area.

the estimate of the moving mass in the model and thus the agreement between the experimental and theoretical frequencies.

\subsection{Energy provided by a dynamic hysteresis}

Besides the frequency, the amplitude of oscillation is relevant to study. The can could stop itself after a few oscillations because of friction with air, with water or with the plate but it is not the case. In the stationary state, the amplitude of oscillation is fixed to a given value for a given set of parameters for the jet and the can. If the initial displacement is lower or higher than this amplitude, the amplitude of the oscillation respectively increases or decreases to reach this value. To investigate this matter, we studied in more details the torque applied by the jet on the can.

The measurements of the torque going one way or the other exhibited a slight difference between the two set of data but it can be attributed to a systematic error along the measurement. Thus, we conclude that there is no static hysteresis that could explain the growing amplitude of oscillation of the can.

Yet, the use of two different coatings showed the importance of the distribution of water around the can in the development of an hysteresis. Indeed, we observed a delay between the moment when the impact point of the jet moves on the other side of the can and when the jet is deflected on the same side. The torque applied on the can obeys consequently to a dynamic hysteresis which develops when the can is moving: the jet of water pushes more the ball one way than the other (Fig. 11a). The graph of the horizontal force is modified because of this hysteresis, the can following the blue line in the graph Figure 11b. The amount of energy gained by the system because of this hysteresis $W$ is thus represented by the red area. The 
application of two different coatings for the same set of parameters proved the importance of the wettability of the surface: when the water hit the non-coated and hydrophobic can, it could not form a uniform layer around the can as it needed to wet a great surface. In this case, the amplitude of oscillation was amplified and the system reached a stationary state with well defined oscillations. But for the coated and more hydrophilic can, where water covered the can symmetrically because of its higher wettability, the amplitude of oscillation decreased and the can stopped itself. This phenomenon that involves surface tension is one of the reason why oscillations were witnessed.

However it might not be the sole explanation. Our force and torques measurements were conducted statically, which is different from the forces applied on the moving can. Because the can is moving up to a speed of $v_{\text {can }}=0.15 \mathrm{~m} / \mathrm{s} \simeq \frac{v_{\text {jet }}}{10}$, the relevant speed of the jet at the impact is the relative one at the surface of the moving can. It may induce an enhanced or hindered deviation of the jet that will influence the motion of the can. We expect the assumption to work well as long as we have $v_{\text {can }} \ll v_{\text {jet }}$ but this is hard to address since measuring dynamic torques experimentally remains difficult. It could be done in simulations.

All in all, the dynamic hysteresis of the torque and maybe to some extent the relative motion of the can compared to the jet are the phenomena responsible for the amplification of the amplitude of oscillation. This gain of energy opposes the loss the system undergoes, such as the friction with air or with the cylinders under the can, the viscosity and the capillary forces due to the presence of water between the can and the cylinders underneath. The contribution of this last phenomenon was showed explicitly by comparing the amplitude of oscillation in absence and in presence of droplets of water under the can. This led to a larger amplitude of oscillation in the first case. The amplitude of motion is thus defined by the equilibrium between the gain and the loss of energy through these various phenomena.

This observation raises the question of the range of parameters required to observe oscillations of the can. Indeed, oscillations are observed only in specific ranges of height or flow of the jet, as well as the curvature of the can. Outside of these ranges, the can is either expelled out of the jet or static under the jet. The can is expelled out of the jet quickly with oscillations amplified too much when the jet reaches a size comparable to the one of the can. Such an observation was also made for a ball levitating over a jet of air [8]. On the other hand, the can found a static equilibrium for important flows or higher speeds of the jet. This is once again likely to be a consequence of a more symmetric distribution of water around the can that prevents it from gaining energy due to the dynamic hysteresis. A qualitative approach by comparing the radius of the jet and the radius of the can is irrelevant in that case, contrary to the case of the expelled ball. In the end, no trivial criterion on the beginning of oscillations has been drawn and it is very likely to depend on a whole domain of parameters for a given can, namely the flow, radius and height of the jet in which persistent oscillations are seen.

\section{Dead ends}

A few other ideas were investigated. Unfortunately it led to unuseful results:

- Fitting of the amplified oscillations and saturation to obtain the new horizontal force applied on the can due to the dynamic hysteresis and the relative motion of the surface of the can compared to the jet.

- Observation of the direct influence of an isolated parameter such as the height of the jet. No interesting trend was found.

- Application of our model to the case of a ball. No cylinder of the size of a ping-pong ball was available to apply its results on a ball. The presence of the plate underneath presumably changed the theoretical model but none was satisfyingly drawn.

\section{Conclusion}

Our work proved that the deviation of the jet along a curved object is responsible for its oscillations under the jet by inducing a restoring force similarly to a spring.

We conducted force and torque measurements which showed that the effective interaction potential between the jet and the cylinder can be modeled by a parabolic potential well. This allowed us to derive a theoretical period of oscillation $T=2 \pi \sqrt{\frac{J_{G}+m R^{2}}{R(k R+\gamma)}}$ that depends on the mass and geometry of the cylinder and on the characteristics of the jet (height, flow and radius). Experimental measurements of the period confirmed that it varies with the square root of the mass. However, a significant error of $20 \%$ is found, which is presumably a consequence of the neglected mass of water of about $3 \mathrm{~g}$ around the cylinder and of the linear approximation of the torque applied on the cylinder.

We also showed that the source of these oscillations is a dynamic hysteresis, which is a consequence of the surface material of the cylinder. There is a moment when the jet hits the can on one side while water is still flowing on the other. This phenomenon leads to a hysteresis of the torque applied on the can that appears only if the cylinder moves, which provides the energy required to observe oscillations. The amplitude of oscillation is eventually a trade-off between the gain in energy and the losses due to friction or capillarity.

Future research will aim at improving our theoretical model and determining more precisely the contribution of the forces included in the total force that we measured. This should also allow us to transpose this study to the two-dimensional problem of the ball.

\section{Supplementary materials}

S1: Ball oscillating under a laminar flow.

S2: A non-coated can undergoing amplified oscillations. 
S3: The same can coated undergoing damped oscillations under the same conditions.

Supplementary materials are available at https: / / emergent-scientist.edp-open.org/10.1051/emsci/ 2020002/olm.

This article is part of the work of the team Université de Lyon for the International Physicists' Tournament. Part of the credits goes to all our teammates, Célia Desgranges, Rémi Faure, Laura Guislain, Gauthier Legrand, Lise Morlet-Decarnin, Hector Mussillon, Arthur Walbecq and Pascal Wang, whose ideas and debates were wise and welcomed. We are also truly grateful to our team leaders Arsène Chemin, Jérémy Ferrand, Nicolas Plihon and Nicolas Taberlet for their help and support during this project.

\section{References}

1. T. Panitz, D. Wasan, AIChE J. 18, 51 (1972)

2. R. Wille, H. Fernholz, J. Fluid Mech. 23, 801 (1965)

3. I. Reba, Sci. Am. 214, 84 (1966)

4. P. Bradshaw, Effect of streamline curvature on turbulent flow, AGARDograph 169 (1973)

5. M. Trancossi, An Overview of Scientific and Technical Literature on Coanda Effect Applied to Nozzles (2011)

6. T. Lòpez-Arias, L.M. Gratton, G. Zendri, S. Oss, Phys. Educ. 46, 146 (2011)

7. R. Friedrich, A.V. Johansson, J. Turbulence 12, N14 (2011)

8. J. Feng, D.D. Joseph, J. Fluid Mech. 315, 367 (1996)

9. D.J. Tritton, Physical Fluid Dynamics (Springer Science \& Business Media, 2012)

Cite this article as: Francis Pagaud and Léa Delance. Driven oscillations of a curved object under a laminar jet of water, Emergent Scientist 5, 1 (2021) 